\title{
Extended Mechanism and Rate-Limiting Step of the Plasminogen Acti- vator Staphylokinase Revealed by Global Kinetic Analysis
}

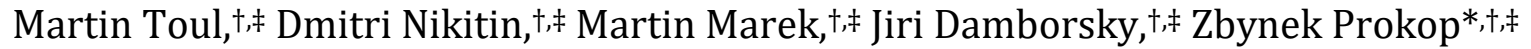

${ }^{\dagger}$ Loschmidt Laboratories, Department of Experimental Biology and Research Centre for Toxic Compounds in the Environment RECETOX, Faculty of Science, Masaryk University, Kamenice 5/C13, 62500 Brno, Czech Republic

‡International Clinical Research Center, St. Anne’s University Hospital Brno, Pekarska 53, 65691 Brno, Czech Republic

\begin{abstract}
The plasminogen activator staphylokinase is a fibrin-specific thrombolytic biomolecule and an attractive target for the development of effective myocardial infarction and stroke therapy. To engineer the protein rationally, a detailed understanding of the biochemical mechanism and limiting steps is essential. Conventional fitting to equations derived based on simplifying approximations may be inaccurate for complex mechanisms like that of staphylokinase. We employed a modern numerical approach of global kinetic data analysis whereby steady-state kinetics and binding affinity datasets were analyzed in parallel. Our approach provided an extended, revised understanding of the staphylokinase mechanism without simplifying approximations and determined the value of turnover number $k_{\text {cat }}$ of $117 \mathrm{~s}^{-1}$ that was 10,000-fold higher than that reported in the literature. The model further showed that the rate-limiting step of the catalytic cycle is binding of staphylokinase to plasmin molecules, which occurs via an induced-fit mechanism. The overall staphylokinase effectivity is further influenced by the formation of an inactive staphylokinase.plasminogen complex. Here, we describe a quick and simplified guide for obtaining reliable estimates of key parameters whose determination is critical to fully understand the staphylokinase catalytic functionality and define rational strategies for its engineering. Our study provides an interesting example of how global numerical analysis of kinetic data can be used to better understand the mechanism and limiting factors of complex biochemical processes.
\end{abstract}

\section{INTRODUCTION}

Cardiovascular diseases, mainly myocardial infarction and ischemic stroke, are the leading causes of morbidity and mortality worldwide responsible for nearly 18 million deaths every year. ${ }^{1}$ Such alarming statistics are a clear motivation to develop efficient thrombolytic treatment strategies. $^{2,3}$ One of the current clinically approved approaches involves using plasminogen activators, i.e., biocatalytic therapeutic molecules with enzymatic activities that help dissolve fibrin clots by generating plasmin (Plm) on the clot surface. ${ }^{4,5}$ However, the efficiency of plasminogen activators is still far from ideal and is often accompanied by side effects and bleeding complications. Thus, there is a need to improve current approaches or find new thrombolytic biocatalysts. ${ }^{2,3}$ Staphylokinase (SAK) is an attractive potential thrombolytic agent owing to its fibrin specificity and low production costs. 6,7 Even though it is immunogenic, it has the potential to be clinically applied, especially in developing countries where cost-effectiveness is prioritized. ${ }^{8-11}$

SAK belongs to the group of so-called indirect plasminogen activators. This means it does not have any biocatalytic activity and cannot initiate fibrinolysis alone. Instead, it follows a complex molecular mechanism (Figure 1) that has been intensively studied.,212-18 SAK forms an equimolar complex with Plm, changing its substrate specificity toward plasminogen (Plg). Consequently, the SAK.Plm complex becomes a typical plasminogen activator capable of Plg-to-Plm catalytic conversion. Finally, generated Plm molecules cleave the fibrin network, resulting in dissolution of fibrin clots to fibrin degradation products. Because two Plm molecules can bind SAK, the terms Plm "partner" and Plm "product" are often used to distinguish between them. 19,20

Even though there have been several attempts to improve the SAK thrombolytic biocatalytic potential by engineering, none of the generated proteins exhibited a significant increase of effectivity. ${ }^{15,21-28}$ This was mainly due to the complex SAK molecular mechanism, which makes it difficult to properly identify the real limiting step, which is critical to be known for rational protein engineering. ${ }^{29,30}$ The major challenge is that the generated Plm "product" can also act as a "partner" and form more of the SAK.Plm complex, continuously changing equilibria without reaching a real steadystate phase. Such a phenomenon makes it impossible to precisely characterize the kinetics of the protein using the conventional approach of initial rate analysis without major approximations. In contrast, global kinetic analysis using numerical integration allows fitting of raw kinetic data directly without any simplifying assumptions. ${ }^{31}$ Thus, even complex multimolecular systems, such as the herein presented SAK case, can be solved with no approximations.

In this study, we systematically collected kinetic data for each step of the SAK molecular mechanism and analyzed them globally using a modern numerical analysis approach. This allowed us to describe an extended mechanism of SAK. Importantly, we identified that there is a substantial underestimation of kinetic parameters when they are calculated by the conventional approach. Based on the newly acquired mechanistic knowledge, we propose an upgraded guide to derive close-to-real estimates of key parameters of SAK kinetics while still using the simple conventional fitting to equations. This method of data processing is available to a wide community and requires no extensive expertise in advanced numerical methods. We believe that the simple upgraded protocol for more detailed SAK characterization will improve its rational engineering toward an optimized therapeutic biocatalyst with a high level of effectivity. In this example, we also demonstrate how the study of other complex biochemical systems could benefit from rigorous global 
kinetic analysis compared to conventional analytical fitting with simplifying approximations. ${ }^{31-33}$

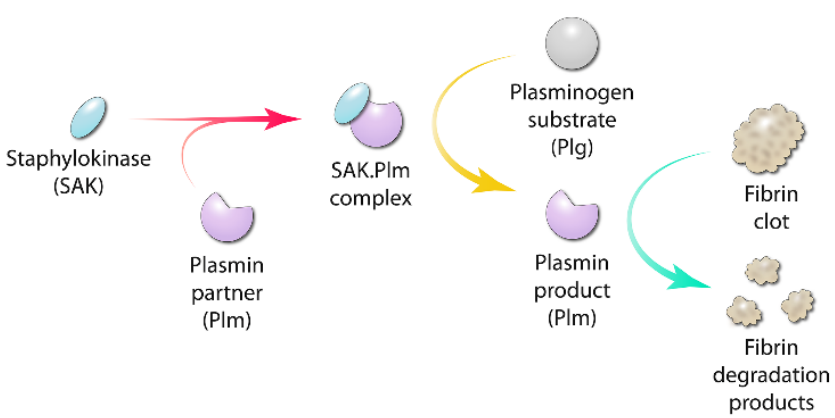

Figure 1. Generally accepted mechanism of staphylokinase. Staphylokinase (SAK) has no enzymatic activity, but it creates an equimolar 1:1 complex with a plasmin (Plm) "partner" to form a catalytically active compound (red arrow). SAK changes the substrate specificity of the Plm "partner" so that the SAK.Plm complex activates the plasminogen (Plg) "substrate" and converts it to additional Plm molecules (yellow arrow). Free generated Plm "products" finally cleave the fibrin network to release degradation products and dissolve fibrin clots (green arrow).

\section{RESULTS}

Biophysical characterization confirms protein quality. After heterologous gene overexpression in E. coli, SAK protein was successfully purified via its His-tag by metal affinity chromatography followed by size-exclusion chromatography, resulting in a purity higher than $95 \%$ (Supplementary Figure S1a) and average yield of $30 \mathrm{mg}$ per one liter of bacterial culture. Subsequent circular dichroism spectroscopy and thermostability measurements confirmed correct folding of the protein (Supplementary Figure S1b) as well as the corresponding melting temperature of $59.4{ }^{\circ} \mathrm{C}$ (Supplementary Figure S1c). Activity measurements using both the standardized fibrin plate assay (Supplementary Figure S1d,e) and indirect chromogenic substrate assay (Supplementary Figure S1f) confirmed that the protein was fully active with a plasminogen activation effectivity comparable to the clinically approved thrombolytic drug alteplase.

Kinetic analysis by conventional approach confirms consistency with previous results. Previous studies have analyzed steady-state kinetics with a premixed solution of SAK and Plm in a 1:1 ratio, assuming that $100 \%$ molecules form the catalytic SAK.Plm complex (Figure 1). The concentration dependence of initial rates then gives a typical Michaelis-Menten relationship from which steadystate kinetic parameters can be determined. However, this approach does not consider that only a fraction of SAK molecules is complexed and, moreover, the equilibrium constantly changes during the reaction due to Plm accumulation.

We applied the same methodology of conventional initial rate analysis to our kinetic data (Figure 2a,b,d,e; Supplementary Table S1) and compared the obtained values with those from the literature. ${ }^{19,24,25,28,34-39}$ Values similar to the reported range (Table 1 ) were obtained for both the turnover number $k_{\text {cat }}\left(0.011 \pm 0.001 \mathrm{~s}^{-1}\right)$ and Michaelis constant $K_{\mathrm{m}}(21 \pm 1 \mu \mathrm{M})$. Such results confirmed the consistency of our kinetic data with those previously reported. a

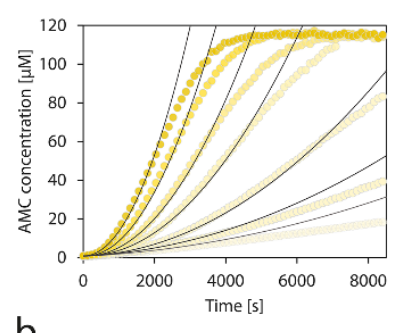

b

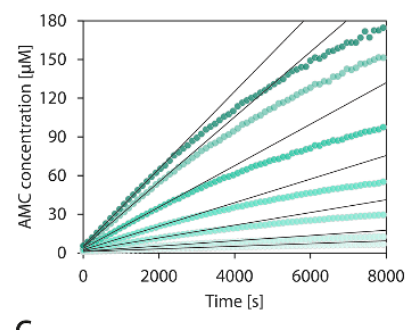

C

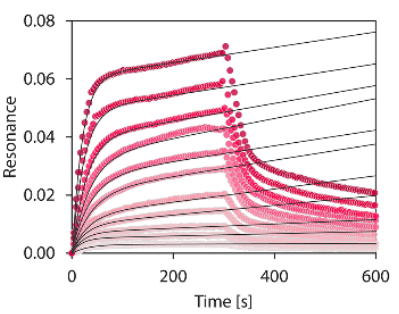

d

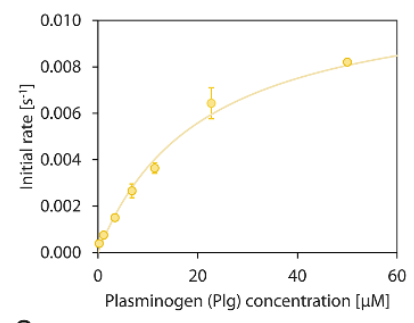

e

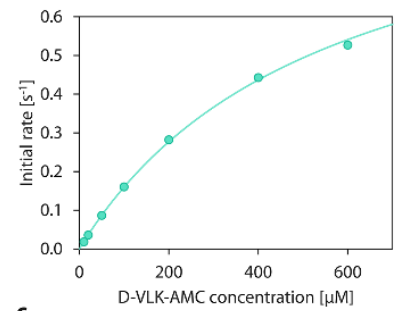

$\mathrm{f}$

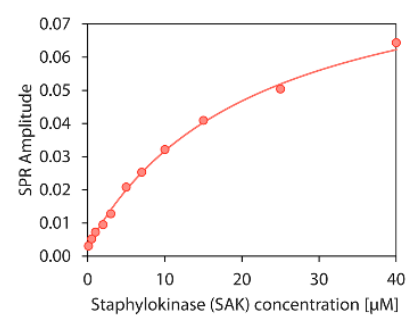

Figure 2. Conventional analytical fitting of staphylokinase kinetic data. The fitting of raw data $(\mathrm{a}-\mathrm{c})$ requires the generation of secondary plots ( $d-f$ ), analyzing concentration dependencies of initial rates and amplitudes separately, losing connections in between them. Analytical fitting was performed for conversion of plasminogen Plg by staphylokinase SAK (yellow curves; a and d), conversion of D-VLK-AMC by plasmin Plm (green curves; $b$ and e), and binding of SAK to immobilized Plm (red curves; $c$ and f). Solid lines represent the best fit. All the experiments were performed in physiological phosphate-buffered saline (PBS) pH 7.4 at $25{ }^{\circ} \mathrm{C}$ in biological duplicates, each in technical triplicates.

The affinity-corrected analysis provides close-toreal values of kinetic parameters. The most significant inaccuracy using the conventional approach is caused by underestimation of the turnover number $k_{\text {cat }}$. This is due to the assumption that all SAK molecules form the catalytic SAK.PIm complex. In reality, a dynamic equilibrium between the free and complexed active forms always exists. If the SAK affinity toward the PIm "partner" is low, only a fraction of the molecules forms the active SAK.Plm complex. Therefore, the actual value of $k_{\text {cat }}$ is proportionally higher.

Using surface plasmon resonance (SPR) affinity measurements (Figure 2c,f), we determined the equilibrium dissociation constant $K_{\mathrm{d}}$ of the SAK.Plm complex of $22 \pm 2 \mu \mathrm{M}$. This was in agreement with previously reported SAK affinity experiments applying analogical experimental conditions. ${ }^{19,40,41}$ Based on this affinity value, we calculated that only $0.03 \%$ of SAK formed the SAK.PIm complex under the experimental steady-state conditions. Hence, the apparent value of $k_{\text {cat }}$ was underestimated by an enormous factor of 3,400 . By simply applying the known SAK.Plm dissociation constant $K_{\mathrm{d}}$, the affinity-corrected value of $k_{\text {cat }}$ can be calculated using Equation 1, where $\mathrm{C}_{\mathrm{SAK}}$ and $\mathrm{C}_{\mathrm{Plm}}$ correspond to 
the concentrations of SAK and Plm applied during the steady-state experiment to pre-form the SAK.PIm complex.

$$
k_{\text {cat }}=k_{\text {cat,app }} \cdot \frac{2 \cdot \mathrm{c}_{\mathrm{SAK}}}{K_{\mathrm{d}}+\mathrm{c}_{\mathrm{SAK}}+\mathrm{c}_{\mathrm{Plm}}-\sqrt{\left(K_{\mathrm{d}}+\mathrm{c}_{\mathrm{SAK}}+\mathrm{c}_{\mathrm{Plm}}\right)^{2}-4 \cdot \mathrm{c}_{\mathrm{SAK}} \cdot \mathrm{c}_{\mathrm{Plm}}}}
$$

This simple adjustment to the conventional data fitting provides results comparable to the advanced global numerical analysis described below (Table 1).

Table 1. Comparison of staphylokinase steady-state kinetic parameters. ${ }^{a}$

$\begin{array}{lllll}\begin{array}{l}\text { Kinetic } \\ \text { parameter }\end{array} & \begin{array}{l}\text { Scientific } \\ \text { literature }\end{array} & \begin{array}{l}\text { Conventional } \\ \text { analysis }\end{array} & \begin{array}{l}\text { Conventional Global } \\ \text { affinity } \\ \text { corrected }\end{array} & \begin{array}{l}\text { kinetic } \\ \text { analysis }\end{array} \\ k_{\text {cat,SAK }\left[\mathrm{s}^{-1}\right]} & 0.01-0.52 & 0.011 \pm 0.01 & 39.5 \pm 0.4 & 117 \pm 9 \\ K_{\mathrm{m}, \mathrm{SAK}}[\mu \mathrm{M}] & 1-20 & 21 \pm 1 & 21 \pm 1 & 56 \pm 4 \\ K_{\mathrm{p}, \mathrm{SAK}}[\mu \mathrm{M}] & \text { n.a. }{ }^{c} & \text { n.a. } .^{c} & \text { n.a. } & >20 \\ K_{\mathrm{d}, \mathrm{PIm}}[\mu \mathrm{M}] & \text { n.a. }{ }^{c} & \text { n.a. }{ }^{c} & 22 \pm 2 & 26 \pm 2 \\ K_{\mathrm{d}, \mathrm{Plg}}[\mu \mathrm{M}] & \text { n.a. }{ }^{c} & \text { n.a. } .^{c} & \text { n.a. }{ }^{c} & 28 \pm 13\end{array}$

${ }^{a}$ The values reported in the literature are compared with the parameters determined herein by different approaches of data analysis. The parameters were derived from kinetic experiments performed in physiological phosphate-buffered saline (PBS) pH 7.4 at $25^{\circ} \mathrm{C}$. $b$ The range is based on previously published results. ${ }^{19,24,25,28,34-39}$ cn.a. $=$ not applicable.

Global kinetic analysis reveals an extended mechanism and rate-limiting step of SAK. As illustrated in Figure 2, the conventional analysis is strictly focused on the initial phases of the reaction and does not consider additional mechanistic information that can be inferred from later kinetic phases. This additional understanding can only be obtained by parallel fitting the full progress curves using numerical data analysis (Figure 3). An extended SAK kinetic mechanism was revealed by applying this approach (Figure 4). The extension provided an accurate quantitative description of the known kinetic steps but also revealed previously undescribed phases. In addition to the basic steadystate kinetic parameters $k_{\text {cat }}$ and $K_{\mathrm{m}}$ (Table 2), the analysis of the entire time course of the kinetic data allowed estimation of the product inhibition constants $K_{\mathrm{p}}$ for both enzymatic reactions (Figure $4-K_{\text {p,SAK }}$ and $K_{\text {p,Plm }}$ ). Their values were comparable to their respective Michaelis constants $K_{\mathrm{m}}$ (Table 2), suggesting that the products could act as potent inhibitors after their accumulation during later stages of the conversion. The newly identified phases of the SAK mechanism included (i) a two-step induced-fit mechanism of SAK binding to Plm (Figure 4 - red pathway), and (ii) an inhibitory effect of Plg "partner" binding on Plg "substrate" activation by SAK (Figure 4 - blue pathway).

The proposed two-step mechanism of Plm binding was based on the SPR data analysis (Figure 3c). The SPR signal exhibited two distinct kinetic phases for both association and dissociation, which could not be explained by a simple one-step binding. Detailed analysis (Supplementary Note S1) revealed that interaction between SAK and Plm occurred via a two-step induced-fit mechanism comprising initial binding of SAK to Plm, followed by conformational change of Plm induced by bound SAK. This result is in accordance with previous mechanistic studies suggesting that binding of SAK induces changes in the substrate specificity of Plm. ${ }^{18,19,42}$ a

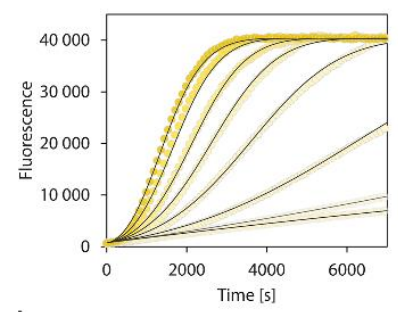

b

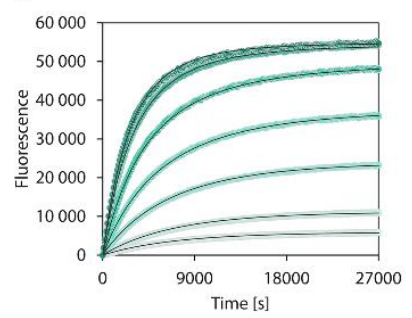

C

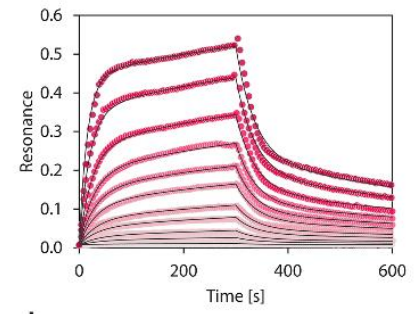

d

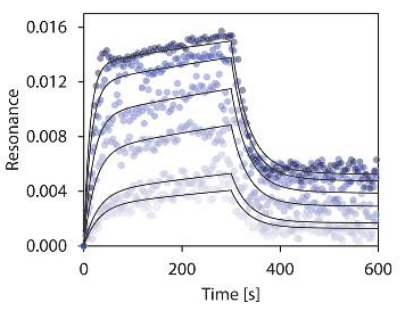

Figure 3. Global numerical analysis of staphylokinase kinetic data. Our approach is based on simultaneous global fitting of the full-progress curves obtained from four different experiments: (a) plasminogen activation and conversion to plasmin by staphylokinase (SAK), (b) D-VLK-AMC conversion to 7amino-4-methylcoumarin (AMC) by plasmin, (c) binding of SAK to plasmin, and (d) binding of SAK to plasminogen. Solid lines represent the best fit. All the experiments were performed in physiological phosphate-buffered saline (PBS) pH 7.4 at $25{ }^{\circ} \mathrm{C}$ in biological duplicates, each in technical triplicates.

The important effect of Plg "partner" binding on the catalysis was derived from the steady-state kinetic curves of Plg conversion by SAK.Plm. When the data were analyzed globally, it was possible to obtain a reasonable fit of the initial phase of Plg activation, but there was an evident slowdown at later stages compared to the expected fit (Supplementary Figure S2a). This deviation could potentially be explained by a product inhibition effect only (Supplementary Figure S3), but the obtained $K_{\mathrm{p}, \mathrm{SAK}}$ value of $0.07 \pm 0.01 \mu \mathrm{M}$ was unrealistically low (Supplementary Table S2); a value 500-fold lower than $K_{\mathrm{m}, \mathrm{SAK}}$ was judged to be unlikely. Thus, this mechanism was assumed to be biochemically irrelevant despite providing a relatively good fit. Another possible cause of the fit deviation could be attributed to the inactive SAK.Plg complex formation. SAK has been reported to bind not only PIm but also Plg molecules as "partners" to form an equimolar complex but the SAK.Plg complex is catalytically inactive and cannot serve as a plasminogen activator.6,14,40 We tested this phenomenon using global kinetic analysis by including an additional dataset obtained from an SPR affinity binding experiment between SAK and Plg (Figure 3d). Plg binding exhibited an induced-fit mechanism similar to that of Plm (Supplementary Note S2). Strikingly, when formation of the SAK.Plg complex was included in the complex kinetic model, the goodness of the global fit was significantly improved and the model fully matched the experimental data (Figure 3 and Supplementary Figure S4). The value of $k_{\text {cat,SAK was }}$ further increased when Plg binding was considered, resulting in an overall underestimation factor of 11,700 when compared to the conventional analysis. A rigorous 

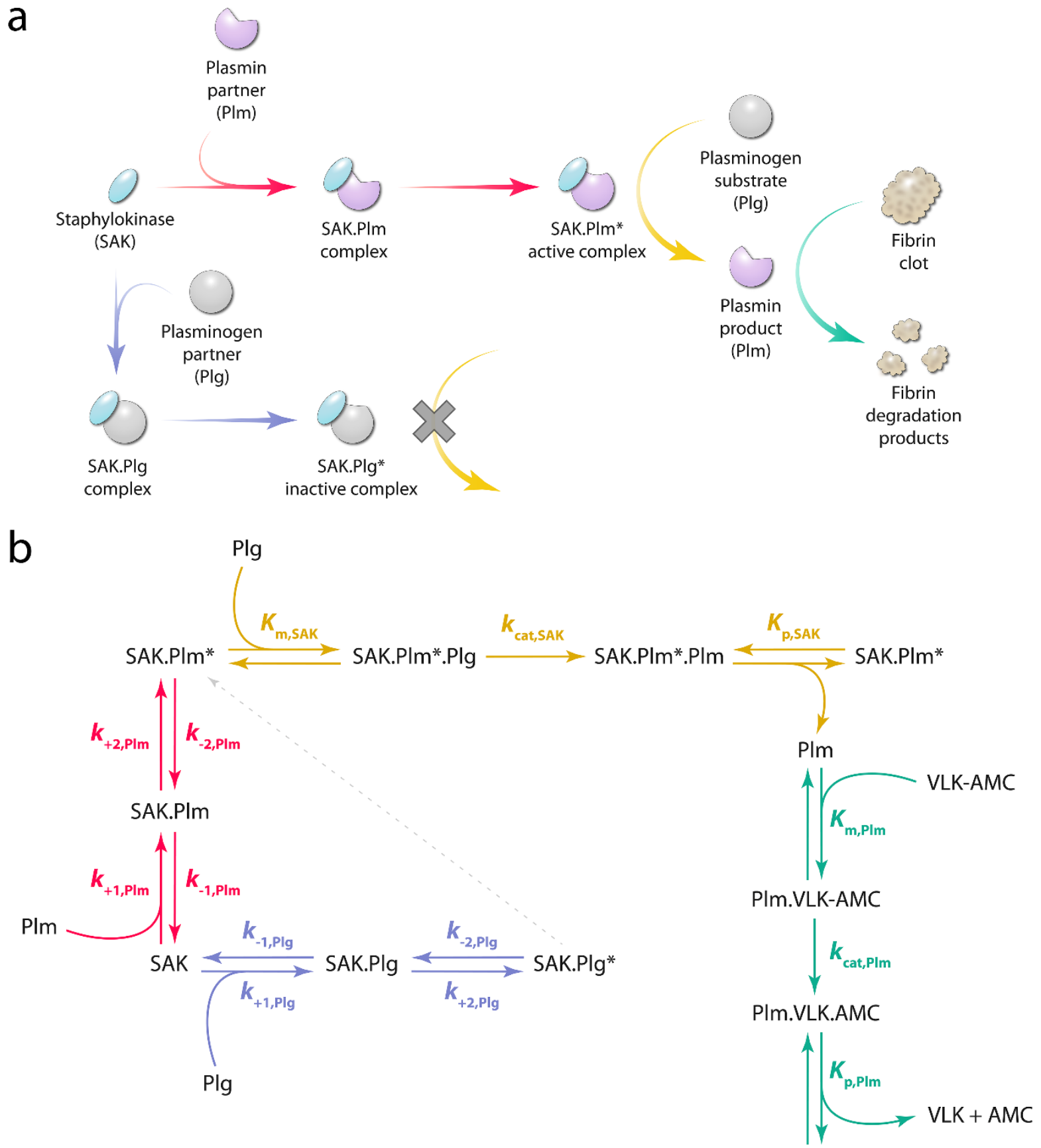

PIm

Figure 4. Extended mechanism of staphylokinase (SAK). (a) Simplified graphical schematic of the mechanism, and (b) detailed kinetic pathway with all analyzed steps and parameters are provided for clarity. Compared to the general mechanism (Figure 1), the extended mechanism newly includes binding of staphylokinase (SAK) to plasminogen (Plg) to form an inactive complex, preventing plasminogen-to-plasmin (Plg-to-Plm) conversion and having a significant effect on the overall effectivity. The mechanism further includes a newly identified two-step induced-fit binding mechanism for both Plm and Plg binding by SAK. SAK.Plg* to SAK.Plm* conversion by Plm, reported previously ${ }^{14}$ and marked with a grey dotted arrow, was not included in the kinetic model because its rate was very slow and not significantly detectable during the experimental time window. Forward and reverse rate constants of the $i$-th step are denoted with the symbols $k_{+\mathrm{i}}$ and $k_{\text {-i, }}$, respectively. The steady-state kinetic parameters $K_{\mathrm{m}}, k_{\text {cat }}$ and $K_{\mathrm{p}}$ correspond to the Michaelis constant, turnover number and product inhibition constant, respectively.

confidence contour analysis (details in Materials and Methods) showed that all the obtained kinetic parameters were well constrained by the data (Table 2, Supplementary Table S3, Supplementary Figure S5) and confirmed the statistical significance of including the event of Plg binding by SAK in the extended SAK mechanism represented in Figure 4.
The simplified guide facilitates quantification of key processes influencing the overall SAK effectivity. To account for the complex biochemical mechanism and additional effects that influence the overall SAK fibrinolytic effectivity, the herein presented global numerical data analysis is the preferred option. However, the advanced kinetic 
methodology requires extensive prior knowledge. Thus, we propose a user-friendly upgrade of the simple conventional data fitting. The upgraded guide (Table 3) allows for easy and reliable quantification of four key processes that influence the overall SAK fibrinolytic effectivity by their respective parameters: (i) plasmin binding, (ii) plasminogen binding, (iii) plasmin selectivity, and (iv) catalytic efficiency. The effect of the key processes is schematically illustrated in Supplementary Figure S6.

Table 2. Kinetic constants describing the extended mechanism of staphylokinase. ${ }^{a}$

$\begin{array}{llll}\text { Parameter } & \text { Value } & \begin{array}{l}\text { Standard } \\ \text { error (S.E.) }\end{array} & \begin{array}{l}\text { Confidence } \\ \text { intervals }\end{array} \\ \text { STAPHYLOKINASE.PLASMIN STEADY-STATE KINETICS } \\ K_{\mathrm{m}, \mathrm{SAK}}[\mu \mathrm{M}] & 56 & 4 & 48-65 \\ k_{\text {cat }, \text { SAK }}\left[\mathrm{s}^{-1}\right] & 117 & 9 & 107-134 \\ K_{\mathrm{p}, \mathrm{SAK}}[\mu \mathrm{M}] & >20 & \text { n.a. }{ }^{c} & >20\end{array}$

PLASMIN STEADY-STATE KINETICS

$\begin{array}{llll}K_{\mathrm{m}, P I m}[\mu \mathrm{M}] & 400 & 30 & 370-450 \\ k_{\mathrm{cat}, P I m}\left[\mathrm{~s}^{-1}\right] & 0.96 & 0.06 & 0.90-1.03 \\ K_{\mathrm{p}, \operatorname{Plm}}[\mu \mathrm{M}] & 310 & 30 & 250-490\end{array}$

PLASMIN BINDING KINETICS

$\begin{array}{llll}k_{+1, \operatorname{Plm}}\left[\mu \mathrm{M}^{-1} . \mathrm{s}^{-1}\right] & 0.000979 & 0.000006 & 0.000919-0.001025 \\ k-1, \operatorname{Plm}\left[\mathrm{s}^{-1}\right] & 0.0252 & 0.0001 & 0.0229-0.0257 \\ k_{+2, \operatorname{Plm}\left[\mathrm{s}^{-1}\right]} & 0.00214 & 0.00008 & 0.00184-0.00219 \\ k_{-2, \operatorname{Plm}\left[\mathrm{s}^{-1}\right]} & 0.0013 & 0.0004 & 0.0010-0.0016\end{array}$

PLASMINOGEN BINDING KINETICS

\begin{tabular}{|c|c|c|c|}
\hline$k_{+1, \operatorname{Plm}}\left[\mu \mathrm{M}^{-1} \cdot \mathrm{s}^{-1}\right]$ & 0.0011 & 0.0005 & $0.0007-0.0014$ \\
\hline$k_{-1, \operatorname{Plm}}\left[\mathrm{s}^{-1}\right]$ & 0.031 & 0.002 & $0.022-0.043$ \\
\hline$k+2, \operatorname{Plm}\left[\mathrm{s}^{-1}\right]$ & 0.0014 & 0.0005 & $0.0007-0.0023$ \\
\hline$k$-2,Plm $\left[\mathrm{s}^{-1}\right]$ & $<0.00016$ & n.a. ${ }^{c}$ & $0-0.00016$ \\
\hline
\end{tabular}

$a$ The parameters and their statistics were obtained by simultaneous global fitting of full-progress curves using numerical integration and by confidence contour analysis with a $\chi^{2}$ threshold of 0.95 . The parameters were derived from kinetic experiments performed in physiological phosphate-buffered saline (PBS) pH 7.4 at $25^{\circ} \mathrm{C} .{ }^{b}$ n.a. $=$ not applicable.

\section{DISCUSSION}

SAK is a promising thrombolytic drug with many advantageous properties, including high fibrin specificity and low production cost. ${ }^{6,7}$ However, its administration is connected with an immune response and its biocatalytic effectivity is not significantly better than current thrombolytic drugs. Thus, there is a need for its improvement. ${ }^{2,3}$ The success of rational protein engineering relies on proper understanding and characterization of the studied system and especially identification of its main limitation. ${ }^{29,30}$ In this study, we addressed the challenge that improper SAK kinetic characterization can cause significant underestimation of the catalytic activity and misleading assumptions that the low activity is the limiting factor of the overall fibrinolytic effectivity. By applying rigorous global kinetic analysis, we showed that the activity of the enzymatic SAK.Plm complex is high, but the inefficient formation of this complex is the main limiting factor.

We validated our kinetic data by first applying a conventional data analysis approach and obtained comparable values of underestimated steady-state kinetic parameters as those reported previously. ${ }^{19,24,25,28,34-39}$ It should be noted that some studies have reported values of $k_{\text {cat }}$ approximately 10-fold higher and out of the range of other consistent results. ${ }^{22,23,26,27,43}$ However, those data were collected at elevated temperatures where exponentially higher rates are expected. Therefore, those results are not in conflict with our observations. Conventional analytical fitting of SPR curves of Plm and Plg binding yielded comparable values of dissociation constants $K_{\mathrm{d}}$ as reported previously for the same experimental conditions, ${ }^{19,40,41}$ confirming the validity of the data used in this study. Similarly, some studies have reported significantly lower values of $K_{\mathrm{d}}^{16,23,26,44-46}$ but these experiments were performed under different conditions, making comparison irrelevant. Most of these studies were based on immobilization of SAK instead of Plm via its amine groups, including the N-terminus. Such arrangement is not optimal since the crystal structure of the SAK. $\mu \mathrm{Plm}$ complex $^{18}$ and mutagenesis functional studies ${ }^{17,40,47}$ have shown that the N-terminal part of SAK must be accessible for its interaction with Plm.

Although many prior reports have focused on the kinetic characterization of SAK and its modified variants, ${ }^{19,22-28,34-}$ 39,43 we were unable to find a single study reporting the analysis of SAK kinetics by a combination of steady-state and binding affinity datasets. Some studies conducted both types of experiments ${ }^{19,23,26,40}$ but did not combine the data to obtain global estimates of kinetic parameters. We employed global numerical analysis of kinetic data to consider not only an initial equilibrium fraction of the active SAK.PIm complex but also assume dynamic changes in this equilibrium due to the ongoing accumulation of Plm during the steady-state kinetic experiment.

Our results showed a significant 10,000-fold underestimation of the turnover number ( $\left.k_{\mathrm{cat}}\right)$ value when the conventional analysis approach was applied. A quick correction to obtain a more realistic value of $k_{\text {cat }}$ can be made by applying a known equilibrium constant of the SAK.Plm complex to the simple equation provided in the Results section. Furthermore, the global data analysis revealed a detailed extended mechanism of SAK, including (i) an induced-fit mechanism for both Plm and Plg binding and (ii) an inhibitory effect of Plg binding on the overall thrombolytic effectivity of SAK. These processes should be considered when characterizing and comparing the effectivities of SAK variants. Conversion of the inactive SAK.Plg complex into the active SAK.Plm complex, reported previously, ${ }^{14}$ was not assumed in our mechanism because it was much slower than other steps of the SAK mechanism and did not alter the data fit. Our detailed analysis with additional pathway steps identified that future modifications of SAK should be focused on improving the Plm selectivity, i.e., either increasing Plm binding or decreasing Plg binding.

While global numerical data analysis without simplifying approximations will always be the preferred option, it is not always easy to use this methodology without extensive prior experience. As an alternative, we propose a simple 
Table 3. Upgraded guide for reliable quantification of SAK kinetics.

\begin{tabular}{|c|c|}
\hline Key parameter ${ }^{a}$ & Determination/calculation \\
\hline Plasmin binding & $\begin{array}{l}\text { Determine } K_{\mathrm{a}, \mathrm{Plm}}\left(=1 / K_{\mathrm{d}, \mathrm{Plm}}\right) \text { of the active SAK.Plm } \\
\text { complex (e.g., by an SPR experiment) to obtain in- } \\
\text { formation about SAK affinity toward Plm. }\end{array}$ \\
\hline Catalytic efficiency & $\begin{array}{l}\text { Calculate the ratio } k_{\mathrm{Cat}, \mathrm{SAK}} / K_{\mathrm{m}, \mathrm{SAK}} \text { using the conven- } \\
\text { tional approach and the affinity-corrected value of } \\
k_{\text {cat }} \text { based on known } K_{\mathrm{d}, \mathrm{Plm}} \text { and Equation } 1 \text {. }\end{array}$ \\
\hline
\end{tabular}

Plasminogen binding Determine $K_{\mathrm{a}, \mathrm{Plg}}\left(=1 / K_{\mathrm{d}, \mathrm{Plg}}\right)$ value of the inactive SAK.Plg complex in an analogical way as for plasmin binding.

Plasmin selectivity
Effect on the overall effectivity

A higher affinity with higher $K_{\mathrm{a}, \mathrm{Plm}}$ leads to an increased fraction of active SAK.Plm, and thus higher overall effectivity.

A higher value of the correctly estimated ratio leads to increased catalytic conversion capability of the pure SAK.Plm complex, and thus higher overall effectivity.

A higher affinity with higher $K_{\text {a,Plg }}$ leads to an increased fraction of inactive SAK.Plg, shifting the equilibrium toward less SAK.Plm, and thus lower overall effectivity.

A higher value of the ratio leads to increased SAK preference toward Plm rather than Plg and formation of more SAK.Plm, and thus higher overall effectivity.

${ }^{a}$ Each parameter quantifying the key process can be estimated in a simplified manner as described in the table without the need for expertise in advanced methods of global kinetic data analysis.

upgrade of the widely used conventional data analysis method to obtain reliable estimates of SAK kinetic parameters. The upgraded guide requires only a few additional steps to determine four key parameters that are critical to fully understand SAK functionality and to correctly interpret the effect of modifications during its engineering.

SAK is only one of many examples of complex multimolecular biochemical systems whose analysis by conventional data fitting might lead to oversimplified results. ${ }^{31-33}$ We believe that our study provides an example of how global numerical analysis of kinetic data can be used to better understand the mechanism and limiting factors of complex biochemical processes.

\section{CONCLUSIONS}

An extended molecular mechanism of SAK was identified by applying a modern global numerical analysis of kinetic data with three major findings: (i) both Plm and Plg "partner" molecules bind SAK via a two-step induced-fit mechanism; (ii) the steady-state kinetic parameters are significantly underestimated if SAK.PIm and SAK.Plg complex formation is not considered; and (iii) the major limitation of SAK biochemistry is the inefficient formation of the catalytically active SAK.Plm complex. Reliable estimates of key parameters can be obtained using the upgraded conventional analysis. The proposed simplified guide may help to easily track and correctly interpret contributions of individual steps to the overall SAK effectivity when designing improved molecules rationally. The results show that future protein engineering of SAK should target improved selectivity of Plm binding and efficiency of SAK.PIm complex formation.

\section{ASSOCIATED CONTENT}

\section{Supporting Information}

Materials and Methods; Supporting Figures and Tables: staphylokinase biophysical characterization and protein quality check, results of alternative tested staphylokinase kinetic mechanisms, additional values of kinetic constants and fitted parameters, results of statistical and constraint analysis of kinetic constants and additional fitted parameters, schematic of the effect of key identified processes on staphylokinase thrombolytic effectivity; Supporting Notes: details of rigorous kinetic analysis of binding mechanisms. This material is available free of charge via the Internet at http://pubs.acs.org.

\section{AUTHOR INFORMATION}

\section{Corresponding Author \\ *zbynek@chemi.muni.cz \\ ORCIDS}

Martin Toul: 0000-0002-3707-6899

Martin Marek: 0000-0001-7220-5644

Jiri Damborsky: 0000-0002-7848-8216

Zbynek Prokop: 0000-0001-9358-4081

Notes

The authors declare no competing financial interest.

\section{ACKNOWLEDGMENT}

The authors would like to express their gratitude to the Ministry of Education of the Czech Republic (INBIO - CZ.02.1.01/0.0/ 0.0/16_026/0008451; TEAMING - CZ.02.1.01/0.0/0.0/ 17_043/0009632), the research infrastructures RECETOX RI LM2018121 and CIISB LM2018127 funded by MEYS CR, the Technology Agency (Permed - TN01000013), and the European Commission (TEAMING - 857560) for financial support. D.N. is a recipient of an individual grant MSCA fellowship (CZ.02.2.69/0.0/0.0/19_074/0016274) and M.T. is supported by a Brno Ph.D. Talent scholarship and an Internal grant agency of Masaryk University IGA MU (MUNI/IGA/1294/2020). M.M. is supported by Masaryk University (MUNI/H/1561/2018).

\section{REFERENCES}

(1) World Health Statistics 2018: Monitoring Health for the SDGs Sustainable Development Goals; World Health Organization, 2018.

(2) Mican, J.; Toul, M.; Bednar, D.; Damborsky, J. Structural Biology and Protein Engineering of Thrombolytics. Comput. Struct. Biotechnol. J. 2019, 17, 917-938. DOI: 10.1016/j.csbj.2019.06.023

(3) Nikitin, D.; Choi, S.; Mican, J.; Toul, M.; Ryu, W.-S.; Damborsky, J.; Mikulik, R.; Kim, D.-E. Development and Testing of 
Thrombolytics in Stroke. J. Stroke 2021, 23 (1), 12-36. DOI: 10.5853/jos.2020.03349

(4) Irigoyen, J. P.; Munoz-Canoves, P.; Montero, L.; Koziczak, M.; Nagamine, Y. The Plasminogen Activator System: Biology and Regulation. Cell. Mol. Life Sci. 1999, 56 (1-2), 104-132. DOI: 10.1007/PL00000615

(5) Baruah, D. B.; Dash, R. N.; Chaudhari, M. R.; Kadam, S. S. Plasminogen Activators: A Comparison. Vascul. Pharmacol. 2006, 44 (1), 1-9. DOI: 10.1016/j.vph.2005.09.003

(6) Lijnen, H. R.; Collen, D. Staphylokinase, a Fibrin-Specific Bacterial Plasminogen Activator. Fibrinolysis 1996, 10 (3), 119126. DOI: $10.1016 /$ S0268-9499(96)80022-1

(7) Collen, D. Staphylokinase: A Potent, Uniquely Fibrin-Selective Thrombolytic Agent. Nat. Med. 1998, 4 (3), 279-284. DOI: 10.1038/nm0398-279

(8) Vanderschueren, S.; Barrios, L.; Kerdsinchai, P.; Van den Heuvel, P.; Hermans, L.; Vrolix, M.; De Man, F.; Benit, E.; Muyldermans, L.; Collen, D.; Van de Werf, F. A Randomized Trial of Recombinant Staphylokinase Versus Alteplase for Coronary Artery Patency in Acute Myocardial Infarction. Circulation 1995, 92 (8), 2044-2049. DOI: 10.1161/01.CIR.92.8.2044

(9) Vanderschueren, S.; Stockx, L.; Wilms, G.; Lacroix, H.; Verhaeghe, R.; Vermylen, J.; Collen, D. Thrombolytic Therapy of Peripheral Arterial Occlusion with Recombinant Staphylokinase. Circulation 1995, 92 (8), 2050-2057. DOI: 10.1161/01.CIR.92.8.2050

(10) Armstrong, P. W.; Burton, J. R.; Palisaitis, D.; Thompson, C. R.; Van de Werf, F.; Rose, B.; Collen, D.; Teo, K. K. Collaborative Angiographic Patency Trial of Recombinant Staphylokinase (CAPTORS). Am. Heart J. 2000, 139 (5), 820-823. DOI: 10.1016/s0002-8703(00)90013-9

(11) Armstrong, P. W.; Burton, J.; Pakola, S.; Molhoek, P. G.; Betriu, A.; Tendera, M.; Bode, C.; Adgey, A. A. J.; Bar, F.; Vahanian, A.; Van de Werf, F. Collaborative Angiographic Patency Trial of Recombinant Staphylokinase (CAPTORS II). Am. Heart J. 2003, 146 (3), 484-488. DOI: 10.1016/S0002-8703(03)00312-0

(12) Kowalska-Loth, B.; Zakrzewski, K. The Activation by Staphylokinase of Human Plasminogen. Acta Biochim. Pol. 1975, 22 (4), 327-339.

(13) Grella, D. K.; Castellino, F. J. Activation of Human Plasminogen by Staphylokinase. Direct Evidence That Preformed Plasmin Is Necessary for Activation to Occur. Blood 1997, 89 (5), 15851589. DOI: $10.1182 /$ blood.V89.5.1585

(14) Collen, D.; Schlott, B.; Engelborghs, Y.; Van Hoef, B.; Hartmann, M.; Lijnen, H. R.; Behnke, D. On the Mechanism of the Activation of Human Plasminogen by Recombinant Staphylokinase. J. Biol. Chem. 1993, 268 (11), 8284-8289. DOI: 10.1016/S00219258(18)53094-5

(15) Schlott, B.; Hartmann, M.; Gührs, K.-H.; Birch-Hirschfeld E.; Gase, A.; Vettermann, S.; Collen, D.; Lijnen, H. R. Functional Properties of Recombinant Staphylokinase Variants Obtained by SiteSpecific Mutagenesis of Methionine-26. Biochim. Biophys. Acta BBA - Protein Struct. Mol. Enzymol. 1994, 1204 (2), 235-242. DOI: 10.1016/0167-4838(94)90013-2

(16) Silence, K.; Hartmann, M.; Guhrs, K.; Gase, A.; Schlott, B.; Collen, D.; Lijnen, H. Structure-Function Relationships in Staphylokinase as Revealed by Clustered Charge to Alanine Mutagenesis. J. Biol. Chem. 1995, 270 (45), 27192-27198. DOI: 10.1074/jbc.270.45.27192

(17) Rajamohan, G.; Dikshit, K. L. Role of the N-Terminal Region of Staphylokinase (SAK): Evidence for the Participation of the $\mathrm{N}$-Terminal Region of SAK in the Enzyme-Substrate Complex Formation. FEBS Lett. 2000, 474 (2-3), 151-158. DOI: 10.1016/S0014-5793(00)01578-7

(18) Parry, M. A.; Fernandez-Catalan, C.; Bergner, A.; Huber, R.; Hopfner, K. P.; Schlott, B.; Gührs, K. H.; Bode, W. The Ternary Microplasmin-Staphylokinase-Microplasmin Complex Is a Proteinase-Cofactor-Substrate Complex in Action. Nat. Struct. Biol. 1998, 5 (10), 917-923. DOI: $10.1038 / 2359$
(19) Singh, S.; Ashish; Dikshit, K. L. Pro42 and Val45 of Staphylokinase Modulate Intermolecular Interactions of His43-Tyr44 Pair and Specificity of Staphylokinase-Plasmin Activator Complex. FEBS Lett. 2012, 586 (6), 653-658. DOI: 10.1016/j.febslet.2012.01.046

(20) Chaudhary, A.; Vasudha, S.; Rajagopal, K.; Komath, S. S.; Garg, N.; Yadav, M.; Mande, S. C.; Sahni, G. Function of the Central Domain of Streptokinase in Substrate Plasminogen Docking and Processing Revealed by Site-Directed Mutagenesis. Protein Sci. 1999, 8 (12), 2791-2805. DOI: 10.1110/ps.8.12.2791

(21) Vanwetswinkel, S.; Plaisance, S.; Zhi-yong, Z.; Vanlinthout, I.; Brepoels, K.; Lasters, I.; Collen, D.; Jespers, L. Pharmacokinetic and Thrombolytic Properties of Cysteine-Linked Polyethylene Glycol Derivatives of Staphylokinase. Blood 2000, 95 (3), 936-942. DOI: 10.1182/blood.V95.3.936.003k20_936_942

(22) Dahiya, M.; Rajamohan, G.; Dikshit, K. L. Enhanced Plasminogen Activation by Staphylokinase in the Presence of Streptokinase $\beta / \beta \gamma$ Domains: Plasminogen Kringles Play a Role. FEBS Lett. 2005, 579 (7), 1565-1572. DOI: 10.1016/j.febslet.2005.01.066

(23) Szemraj, J.; Walkowiak, B.; Kawecka, I.; Janiszewska, G.; Buczko, W.; Bartkowiak, J.; Chabielska, E. A New Recombinant Thrombolytic and Antithrombotic Agent with Higher Fibrin Affinity - A Staphylokinase Variant. I. In Vitro Study. J. Thromb. Haemost. 2005, 3 (10), 2156-2165. DOI: $10.1111 /$ j.15387836.2005.01480.x

(24) Chen, H.; Mo, W.; Su, H.; Zhang, Y.; Song, H. Characterization of a Novel Bifunctional Mutant of Staphylokinase with PlateletTargeted Thrombolysis and Antiplatelet Aggregation Activities. BMC Mol. Biol. 2007, 8 (1), 88. DOI: 10.1186/1471-2199-8-88

(25) Chen, H.; Mo, W.; Zhang, Y.; Su, H.; Ma, J.; Yao, R.; Zhang, S.; Ge, J.; Song, H. Functional Properties of a Novel Mutant of Staphylokinase with Platelet-Targeted Fibrinolysis and Antiplatelet Aggregation Activities. Eur. J. Pharmacol. 2007, 566 (1), 137-144. DOI: 10.1016/j.ejphar.2007.03.010

(26) Kowalski, M.; Brown, G.; Bieniasz, M.; Oszajca, K.; Chabielska, E.; Pietras, T.; Szemraj, Z.; Makandjou-Ola, E.; Bartkowiak, J.; Szemraj, J. Cloning and Expression of a New Recombinant Thrombolytic and Anthithrombotic Agent - A Staphylokinase Variant. Acta Biochim. Pol. 2009, 56 (1). DOI: 10.18388/abp.2009_2515

(27) Pulicherla, K. K.; Kumar, A.; Gadupudi, G. S.; Kotra, S. R.; Sambasiva Rao, K. R. S. In Vitro Characterization of a Multifunctional Staphylokinase Variant with Reduced Reocclusion, Produced from Salt Inducible E. coli GJ1158. BioMed Res. Int. 2013, 2013, e297305. DOI: 10.1155/2013/297305

(28) Bhando, T.; Singh, S.; Hade, M. D.; Kaur, J.; Dikshit, K. L. Integration of VEK-30 Peptide Enhances Fibrinolytic Properties of Staphylokinase. Biotechnol. Appl. Biochem. 2020, 68 (2). DOI: 10.1002/bab.1912

(29) Lutz, S. Beyond Directed Evolution - Semi-Rational Protein Engineering and Design. Curr. Opin. Biotechnol. 2010, 21 (6), 734-743. DOI: 10.1016/j.copbio.2010.08.011

(30) Singh, R. K.; Lee, J.-K.; Selvaraj, C.; Singh, R.; Li, J.; Kim, S.Y.; Kalia, V. C. Protein Engineering Approaches in the Post-Genomic Era. Curr. Protein Pept. Sci. 2018, 19 (1), 5-15. DOI: $10.2174 / 1389203718666161117114243$

(31) Johnson, K. A. A Century of Enzyme Kinetic Analysis, 1913 to 2013. FEBS Lett. 2013, 587 (17), 2753-2766. DOI: 10.1016/j.febslet.2013.07.012

(32) Morton, T. A.; Myszka, D. G.; Chaiken, I. M. Interpreting Complex Binding Kinetics from Optical Biosensors: A Comparison of Analysis by Linearization, the Integrated Rate Equation, and $\mathrm{Nu}-$ merical Integration. Anal. Biochem. 1995, 227 (1), 176-185. DOI: 10.1006/abio.1995.1268

(33) Hay, S. Extracting Kinetic Isotope Effects From a Global Analysis of Reaction Progress Curves. In Methods in Enzymology; Elsevier, 2017; pp 85-111. DOI: 10.1016/bs.mie.2017.06.041

(34) Dahiya, M.; Singh, S.; Rajamohan, G.; Sethi, D.; Ashish; Dikshit, K. L. Intermolecular Interactions in Staphylokinase- 
Plasmin(ogen) Bimolecular Complex: Function of His43 and Tyr44 FEBS Lett. 2011, 585 (12), 1814-1820. DOI: 10.1016/j.febslet.2011.04.030

(35) Okada, K.; Yuasa, H.; Hagiya, Y.; Fukao, H.; Ueshima, S.; Matsuo, O. Kinetic Analysis of Plasminogen Activation by Staphylokinase/Plasminogen Complex in the Presence of Fibrin. Thromb. Res. 1994, 76 (2), 181-191. DOI: 10.1016/0049-3848(94)90188-0

(36) Shibata, H.; Nagaoka, M.; Sakai, M.; Sawada, H.; Watanabe, T.; Yokokura, T. Kinetic Studies on the Plasminogen Activation by the Staphylokinase-Plasmin Complex. J. Biochem. (Tokyo) 1994, 115 (4), 738-742. DOI: 10.1093/oxfordjournals.jbchem.a124404

(37) Aisina, R. B.; Mukhametova, L. I.; Gulin, D. A.; Gershkovich, K. B.; Varfolomeyev, S. D. Streptokinase and Staphylokinase: Differences in the Kinetics and Mechanism of Their Interaction with Plasminogen, Inhibitors, and Fibrin. Russ. J. Bioorganic Chem. 2015, 41 (5), 506-517. DOI: 10.1134/S1068162015050039

(38) Aisina, R.; Mukhametova, L.; Gershkovich, K.; Varfolomeyev, S. The Role of Carbohydrate Side Chains of Plasminogen in Its Activation by Staphylokinase. Biochim. Biophys. Acta BBA - Gen. Subj. 2005, 1725 (3), 370-376. DOI: 10.1016/j.bbagen.2005.07.007

(39) Okada, K.; Ueshima, S.; Takaishi, T.; Yuasa, H.; Fukao, H.; Matsuo, O. Effects of Fibrin and $\alpha 2$-Antiplasmin on Plasminogen Activation by Staphylokinase. Am. J. Hematol. 1996, 53 (3), 151157. DOI: 10.1002/(SICI)1096-8652(199611)53:3<151::AID$\mathrm{AJH} 1>3.0 . \mathrm{CO} ; 2-\mathrm{Y}$

(40) Schlott, B.; Gührs, K.-H.; Hartmann, M.; Röcker, A.; Collen, D. $\mathrm{NH}_{2}$-Terminal Structural Motifs in Staphylokinase Required for Plasminogen Activation. J. Biol. Chem. 1998, 273 (35), 2234622350. DOI: $10.1074 /$ jbc.273.35.22346
(41) Schlott, B.; Gührs, K.-H.; Hartmann, M.; Röcker, A.; Collen, D. Staphylokinase Requires $\mathrm{NH}_{2}$-Terminal Proteolysis for Plasminogen Activation. J. Biol. Chem. 1997, 272 (9), 6067-6072. DOI: 10.1074/jbc.272.9.6067

(42) Arai, K.; Mimuro, J.; Madoiwa, S.; Matsuda, M.; Sako, T.; Sakata, Y. Effect of Staphylokinase Concentration on Plasminogen Activation. Biochim. Biophys. Acta BBA - Gen. Subj. 1995, 1245 (1), 69-75. DOI: 10.1016/0304-4165(95)00064-I

(43) Rajamohan, G.; Dahiya, M.; Mande, S. C.; Dikshit, K. L. Function of the 90-Loop (Thr90-Glu100) Region of Staphylokinase in Plasminogen Activation Probed through Site-Directed Mutagenesis and Loop Deletion. Biochem. J. 2002, 365 (Pt 2), 379-389. DOI: 10.1042/BJ20011647

(44) Jespers, L.; Van Herzeele, N.; Lijnen, H. R.; Van Hoef, B.; De Maeyer, M.; Collen, D.; Lasters, I. Arginine 719 in Human Plasminogen Mediates Formation of the Staphylokinase:Plasmin Activator Complex. Biochemistry 1998, 37 (18), 6380-6386. DOI: 10.1021/bi972807i

(45) Rodríguez, P.; Collen, D.; Lijnen, H. R. Binding of Streptokinase and Staphylokinase to Plasminogen. Fibrinolysis 1995, 9 (5), 298-303. DOI: 10.1016/S0268-9499(95)80019-0

(46) Lijnen, H. R.; Cock, F. D.; Hoef, B. V.; Schlott, B.; Collen, D. Characterization of the Interaction Between Plasminogen and Staphylokinase. Eur. J. Biochem. 1994, 224 (1), 143-149. DOI: 10.1111/j.1432-1033.1994.tb20005.x

(47) Gase, A.; Hartmann, M.; Guhrs, K. H.; Rocker, A.; Collen, D.; Behnke, D.; Schlott, B. Functional Significance of $\mathrm{NH}_{2-}$ and $\mathrm{COOH}-T e r m i n a l$ Regions of Staphylokinase in Plasminogen Activation. Thromb. Haemost. 1996, 76 (5), 755-760. 
Authors are required to submit a graphic entry for the Table of Contents (TOC) that, in conjunction with the manuscript title, should give the reader a representative idea of one of the following: A key structure, reaction, equation, concept, or theorem, etc., that is discussed in the manuscript. Consult the journal's Instructions for Authors for TOC graphic specifications.

\section{Insert Table of Contents artwork here}

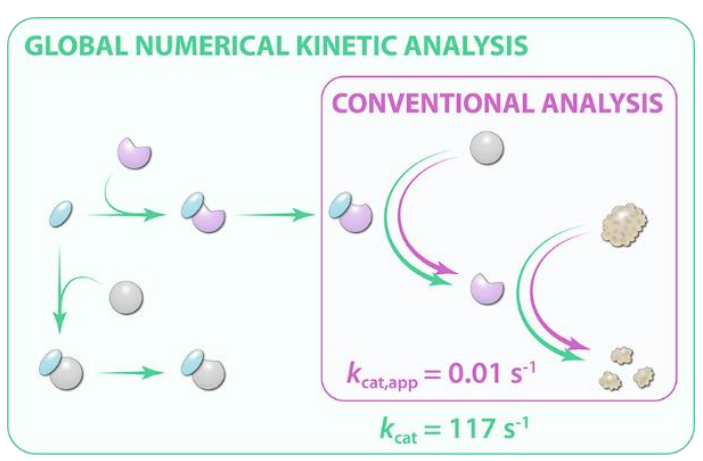

